\title{
A periodic SEIRS epidemic model with a time-dependent latent period
}

\author{
Fuxiang $\mathrm{Li}^{1} \cdot$ Xiao-Qiang Zhao ${ }^{1}$
}

Received: 28 February 2018 / Revised: 19 July 2018 / Published online: 4 January 2019

(c) Springer-Verlag GmbH Germany, part of Springer Nature 2019

\begin{abstract}
Many infectious diseases have seasonal trends and exhibit variable periods of peak seasonality. Understanding the population dynamics due to seasonal changes becomes very important for predicting and controlling disease transmission risks. In order to investigate the impact of time-dependent delays on disease control, we propose an SEIRS epidemic model with a periodic latent period. We introduce the basic reproduction ratio $R_{0}$ for this model and establish a threshold type result on its global dynamics in terms of $R_{0}$. More precisely, we show that the disease-free periodic solution is globally attractive if $R_{0}<1$; while the system admits a positive periodic solution and the disease is uniformly persistent if $R_{0}>1$. Numerical simulations are also carried out to illustrate the analytic results. In addition, we find that the use of the temporal average of the periodic delay may underestimate or overestimate the real value of $R_{0}$.
\end{abstract}

Keywords Periodic SEIRS model · Time-dependent latent period · Basic reproduction ratio $\cdot$ Periodic solution $\cdot$ Uniform persistence

Mathematics Subject Classification 34K13 · 37N25 · 92D30

\section{Introduction}

Mathematical models provide powerful tools to explain and predict the spread of infectious diseases, and to test control strategies. One of the earliest models in epidemiology was introduced in 1927 (see Kermack and McKendrick 1991). Since then, numerous

Fuxiang Li

fuxiangl@mun.ca

Xiao-Qiang Zhao

zhao@mun.ca

1 Department of Mathematics and Statistics, Memorial University of Newfoundland, St. John's, NL A1C 5S7, Canada 
mathematical models for infectious diseases have been described by autonomous systems of differential equations, e.g., the dynamics of SIS, SIR, SIRS, SEI, SEIR and SEIRS epidemic models was intensively investigated in the last few decades (see, e.g., Anderson and May 1979; Cooke and van den Driessche 1996; Gao et al. 1995; Hethcote 1976; Huang et al. 2010; Kermack and McKendrick 1932; Ma et al. 2004).

In fact, many diseases show seasonal behaviors (see, e.g., Altizer et al. 2006; Dowell 2001; Fisman 2007; Grassly and Fraser 2006). From Fares (2011), Meyer et al. (1983), Grassly and Fraser (2006), London and Yorke (1973) and Purse et al. (2005), we have known that measles, diphtheria, chickenpox, tuberculosis, influenza, cholera and malaria in humans, bluetongue in cattle and sheep, viral haemorrhagic septicaemia and furunculosis in fish, are recognized as having seasonal trends and show variable periods of peak seasonality. The causes of seasonal patterns for different diseases are complex, such as temperature, humidity, photoperiod, host aggregation and resource availability. For example, there is a strong relationship between the seasonal outbreaks of meningococcal meningitis disease and climate, e.g., wind speed and low absolute humidity affect respiratory transmission (see Sultan et al. 2005). As mentioned in Dowell et al. (2003), the seasonal patterns of invasive pneumococcal disease in humans correlate with the photoperiod-dependent variation in host susceptibility and fall aggregation among school children. In Vaidya and Wahl (2015), a mathematical model of low pathogenic avian influenza dynamics that includes both time-varying environmental effects and seasonal migration was considered. In addition, unlike warm-blooded animals, temperature has a particularly important influence on fish diseases in areas where there is a wide amplitude in daily and seasonal temperature changes because it affects the rate of metabolism, immunologic response, reproduction, amount of oxygen dissolved in water, biological oxygen demand, toxicity of pollutants, and growth of fish pathogens and parasites (see, e.g., Meyer et al. 1983; Snieszko 1974).

Annual changes in host and parasite biology can generate outbreaks that occur around the same time each year, and there is the growing awareness that seasonality can cause population fluctuations ranging from annual cycles to multiyear oscillations, and even chaotic dynamics (see, e.g., Altizer et al. 2006; Aron and Schwartz 1984; Greenman et al. 2004). It thus becomes natural to model these diseases by incorporating periodic variations into epidemic models. For example, Liu et al. (2010) studied the global dynamics of a non-autonomous SEIR system for tuberculosis with seasonality by introducing a possible seasonal variation in pulmonary tuberculosis. Towers et al. (2011) proposed an SIR epidemic model with periodic transmission rate to assess the efficacy of control strategies via antiviral drug treatment during an outbreak of pandemic influenza. Zhang and Teng (2007) considered a non-autonomous SEIRS epidemic model and established some sufficient conditions for the permanence and extinction of the disease. Later, Nakata and Kuniya (2010) improved the results in a periodic environment.

Cooke and van den Driessche (1996) proposed an SEIRS epidemic model with two time delays. Since then, a number of the dynamic behaviors of SEIRS epidemic models with constant delays have been studied (see, e.g., Jiao et al. 2008; Qi and Cui 2013; Wang 2002; Zhao 2017a). Meanwhile, many population models with timedependent delays have been developed (see, e.g., Beck-Johnson et al. 2013; Lou and Zhao 2017; Omori and Adams 2011; Wang and Zhao 2017b). Recently, Lou and Zhao 
(2017) proposed a host-macroparasite model with seasonal developmental durations and time-dependent delays, and studied the global dynamics by introducing a periodic semiflow on a suitably chosen phase space. Wang and Zhao (2017b) studied the global dynamics of a malaria transmission model with a time-dependent incubation period by using the same theoretical approach as in Lou and Zhao (2017). Indeed, the latent (or incubation) periods of quite a few diseases are related to seasonal changes. For some vector-borne diseases, there is considerable evidence to show that the extrinsic incubation period of the parasite is always sensitive to temperature. Similarly, for the directly transmitted diseases, the length of the latent period depends not only on the strength of the individual's immune system, but also on the climate changes, especially for the aquatic animal and plant disease epidemics (see, e.g., Meyer et al. 1983; Groberg et al. 1978; Lovell et al. 2004; Omori and Adams 2011). For example, the incubation period for fish furunculosis ranges probably from 2 to 4 days. However, at lower temperatures, the incubation period may be extended by several weeks (see Groberg et al. 1978). Omori and Adams (2011) developed a mathematical model with time-dependent delays to analyse the effect of seasonal temperature cycles on koi herpes virus (KHV) in common carp due to the time delays depending on water temperature. Therefore, it is more reasonable to incorporate this seasonally forced latent period into the disease transmission models.

Motivated by the above works, the purpose of this paper is to develop a class of periodic SEIRS epidemic models that, for the first time, incorporates the timedependent latent period. We will use the theoretical approach developed in Lou and Zhao (2017) and the theory of uniform persistence for periodic semiflows to study our model system.

The organization of the paper is as follows. In Sect. 2, we formulate the model and give the underlying assumptions. In Sect. 3, we introduce the basic reproduction ratio $R_{0}$ for the model system and show that $R_{0}$ acts as a threshold parameter for the uniform persistence and global extinction of the disease. In Sect. 4, some numerical simulations are presented to illustrate the main results.

\section{Model formulation}

In order to formulate the model, we first consider a classical SIRS epidemic model in a population. Let $N(t)$ be the total population number at time $t$ which is divided into three classes: susceptible population, infectious population, and recovered (or removed) population (i.e., who have been infected and then removed from the possibility of infection through the temporary immunity). Let $S(t), I(t)$, and $R(t)$ be the total number of the susceptible, infectious, and recovered (or removed) populations at time $t$, respectively. Anderson and May (1979) proposed the following famous SIRS model:

$$
\begin{aligned}
\frac{d S(t)}{d t} & =\Lambda-\beta S(t) I(t)-\mu S(t)+\alpha R(t), \\
\frac{d I(t)}{d t} & =\beta S(t) I(t)-(\mu+d+\gamma) I(t), \\
\frac{d R(t)}{d t} & =\gamma I(t)-\mu R(t)-\alpha R(t) .
\end{aligned}
$$


Here the constant $\Lambda$ is the recruitment rate, $\beta$ is the disease transmission coefficient, $\mu$ is the natural death rate of the population, $d$ is the disease-induced death rate, $\gamma$ is the recovery rate of the infectious population, and $\alpha$ is the lose of immunity rate.

In fact, upon infection, the susceptible individuals become exposed for some diseases (e.g., tuberculosis, influenza, measles, KHV epidemiology); that is, infected but not yet infective. These individuals remain in the exposed class for a certain latent period before becoming infective. Thus it is natural to introduce a latent delay into system (1) and consider an SEIRS epidemic model. Let $E(t)$ be the total number of the exposed population at time $t$, and $M(t)$ be the number of newly occurred infectious population per unit time at time $t$. We assume that the latent period of the disease is time-periodic due to the seasonal weather changes, denoted by $\tau(t)$. Motivated by Liu et al. (2010), Lou and Zhao (2017), Mateus and Silva (2017), Nakata and Kuniya (2010), Omori and Adams (2011), Towers et al. (2011), Wang and Zhao (2017b) and Zhang and Teng (2007), we propose the following evolution system with general incidence rate:

$$
\begin{aligned}
& \frac{d S(t)}{d t}=\Lambda(t)-f(t, S(t), I(t))-\mu(t) S(t)+\alpha(t) R(t), \\
& \frac{d E(t)}{d t}=f(t, S(t), I(t))-\mu(t) E(t)-M(t), \\
& \frac{d I(t)}{d t}=M(t)-(\mu(t)+d(t)+\gamma(t)) I(t), \\
& \frac{d R(t)}{d t}=\gamma(t) I(t)-\mu(t) R(t)-\alpha(t) R(t),
\end{aligned}
$$

where $\Lambda(t), \mu(t), \alpha(t), d(t)$, and $\gamma(t)$ have the same biological meanings as $\Lambda, \mu$, $\alpha, d$, and $\gamma$ in system (1), respectively. The incidence function $f(t, S, I)$ depends on time $t$ and variables $S$ and $I$. In the following, we will use the arguments similar to those in Omori and Adams (2011) and Nisbet and Gurney (1983).

Let $q$ represent the development level of infection such that $q$ increases at a timedependent rate $\kappa(t)$, and assume that $q=q_{E}=0$ at the transition from $S$ to $E$, and $q=q_{I}$ at the transition from $E$ to $I$. The variable $q$ describes how complete the latent stage is. Let $\rho(q, t)$ be the density of individuals with infection development level $q$ at time $t$. Then $M(t)=\kappa(t) \rho\left(q_{I}, t\right)$.

Let $J(q, t)$ be the flux, in the direction of increasing $q$, of individuals with infection development level $q$ at time $t$. Thus, we have the following equation (see, e.g., Kot 2001)

$$
\frac{\partial \rho(q, t)}{\partial t}=-\frac{\partial J}{\partial q}-\mu(t) \rho .
$$

Since $J(q, t)=\kappa(t) \rho(q, t)$, we have

$$
\frac{\partial \rho(q, t)}{\partial t}=-\frac{\partial[\kappa(t) \rho(q, t)]}{\partial q}-\mu(t) \rho(q, t) .
$$


For the state $E$, system (3) has the boundary condition

$$
\rho\left(q_{E}, t\right)=\frac{f(t, S(t), I(t))}{\kappa(t)} .
$$

In order to solve equation (3) with the above boundary condition, we introduce a new variable

$$
\eta=h(t):=q_{E}+\int_{0}^{t} \kappa(s) d s .
$$

Let $h^{-1}(\eta)$ be the inverse function of $h(t)$, and denote

$$
\hat{\rho}(q, \eta)=\rho\left(q, h^{-1}(\eta)\right), \hat{\mu}(\eta)=\mu\left(h^{-1}(\eta)\right), \hat{\kappa}(\eta)=\kappa\left(h^{-1}(\eta)\right) .
$$

From (3), we have

$$
\frac{\partial \hat{\rho}(q, \eta)}{\partial \eta}=-\frac{\partial \hat{\rho}(q, \eta)}{\partial q}-\frac{\hat{\mu}(\eta)}{\hat{\kappa}(\eta)} \hat{\rho}(q, \eta) .
$$

Let $V(s)=\hat{\rho}(s+q-\eta, s)$. Then

$$
\frac{d V(s)}{d s}=-\frac{\hat{\mu}(\eta)}{\hat{\kappa}(\eta)} V(s) .
$$

Since $\eta-\left(q-q_{E}\right) \leq \eta$, we have

$$
V(\eta)=V\left(\eta-\left(q-q_{E}\right)\right) e^{-\int_{\eta-\left(q-q_{E}\right.}^{\eta} \frac{\hat{\mu}(s)}{\hat{\kappa}(s)} d s} .
$$

Then

$$
\hat{\rho}(q, \eta)=\hat{\rho}\left(q_{E}, \eta-\left(q-q_{E}\right)\right) e^{-\int_{\eta-\left(q-q_{E}\right)}^{\eta} \frac{\hat{\mu}(s)}{\hat{\kappa}(s)} d s} .
$$

Let $\tau(q, t)$ be the time taken to grow from infection development level $q_{E}$ to level $q$ by a individual who arrives at infection development level $q$ at time $t$. Since $d q / d t=\kappa(t)$, we have

$$
q-q_{E}=\int_{t-\tau(q, t)}^{t} \kappa(s) d s
$$

and hence,

$h(t-\tau(q, t))=q_{E}+\int_{0}^{t-\tau(q, t)} \kappa(s) d s=h(t)-\int_{t-\tau(q, t)}^{t} \kappa(s) d s=h(t)-q+q_{E}$. 
Letting $s=h(r)$, we then obtain

$$
\int_{\eta-\left(q-q_{E}\right)}^{\eta} \frac{\hat{\mu}(s)}{\hat{\kappa}(s)} d s=\int_{t-\tau(q, t)}^{t} \mu(r) d r .
$$

It follows that

$$
\begin{aligned}
\rho(q, t) & =\hat{\rho}(q, h(t)) \\
& =\rho\left(q_{E}, t-\tau(q, t)\right) e^{-\int_{t-\tau(q, t)}^{t} \mu(r) d r} \\
& =\frac{f(t-\tau(q, t), S(t-\tau(q, t)), I(t-\tau(q, t)))}{\kappa(t-\tau(q, t))} e^{-\int_{t-\tau(q, t)}^{t} \mu(r) d r} .
\end{aligned}
$$

Let $\tau(t)=\tau\left(q_{I}, t\right)$. Then we have

$$
\kappa(t) \rho\left(q_{I}, t\right)=f(t-\tau(t), S(t-\tau(t)), I(t-\tau(t))) \frac{\kappa(t)}{\kappa(t-\tau(t))} e^{-\int_{t-\tau(t)}^{t} \mu(r) d r} .
$$

Letting $q=q_{I}$ and $q_{E}=0$ in (4), we then obtain

$$
q_{I}=\int_{t-\tau(t)}^{t} \kappa(s) d s
$$

where $\kappa(s)$ is $\omega$-periodic in $s$. Clearly, $\tau(t)$ is an implicitly defined function of $t$. The periodicity of $\kappa(s)$ in $s$ implies the periodicity of the delay $\tau(t)$ in time variable $t$. Taking the derivative with respect to $t$, we have

$$
1-\tau^{\prime}(t)=\frac{\kappa(t)}{\kappa(t-\tau(t))}>0 .
$$

Substituting $M(t)=\kappa(t) \rho\left(q_{I}, t\right)$ into system (2), we obtain the following system:

$$
\begin{aligned}
\frac{d S(t)}{d t}= & \Lambda(t)-f(t, S(t), I(t))-\mu(t) S(t)+\alpha(t) R(t), \\
\frac{d E(t)}{d t}= & f(t, S(t), I(t))-\mu(t) E(t)-\left(1-\tau^{\prime}(t)\right) e^{-\int_{t-\tau(t)}^{t} \mu(r) d r} f(t-\tau(t), \\
& S(t-\tau(t)), I(t-\tau(t))), \\
\frac{d I(t)}{d t}= & \left(1-\tau^{\prime}(t)\right) e^{-\int_{t-\tau(t)}^{t} \mu(r) d r} f(t-\tau(t), S(t-\tau(t)), \\
& I(t-\tau(t)))-(\mu(t)+d(t)+\gamma(t)) I(t), \\
\frac{d R(t)}{d t}= & \gamma(t) I(t)-\mu(t) R(t)-\alpha(t) R(t) .
\end{aligned}
$$


We assume that $f(t, S, I)$ and all these time-dependent coefficients are $\omega$-periodic in $t$ for some real number $\omega>0$. Then it is easy to see that the function

$$
a(t):=e^{-\int_{t-\tau(t)}^{t} \mu(r) d r}
$$

is also $\omega$-periodic, and system (5) is an $\omega$-periodic functional differential system.

In view of biological meanings, we should impose the following compatibility condition:

$$
E(0)=\int_{-\tau(0)}^{0} e^{-\int_{s}^{0} \mu(r) d r} f(s, S(s), I(s)) d s .
$$

By the uniqueness of solutions, we then have

$$
E(t)=\int_{t-\tau(t)}^{t} e^{-\int_{s}^{t} \mu(r) d r} f(s, S(s), I(s)) d s .
$$

To study the evolution dynamics of system (5), we make the following assumptions:

(A1) $\Lambda(t), \mu(t), \alpha(t), d(t)$, and $\gamma(t)$ are all non-negative and continuous functions with $\Lambda(t)>0, \int_{0}^{\omega} \mu(t) d t>0$, and $\int_{0}^{\omega} \gamma(t) d t>0$;

(A2) $f(t, S, I)$ is a $C^{1}$-function with the following properties:

(i) $f(t, S, 0) \equiv 0, f(t, 0, I) \equiv 0$, and $\frac{\partial f(t, S, 0)}{\partial I}$ is positive and non-decreasing for all $S>0$.

(ii) $\frac{\partial f(t, S, I)}{\partial S} \geq 0$ and $f(t, S, I) \leq \frac{\partial f(t, S, 0)}{\partial I} I$ for all $(t, S, I) \in \mathbb{R} \times \mathbb{R}_{+}^{2}$. $\geq 0$.

A prototypical example for incidence function is $f(t, S, I)=\frac{\beta(t) S I}{1+\varepsilon(t) I}$ with $\varepsilon(t)$

\section{Threshold dynamics}

In this section, we first introduce the basic reproduction ratio $R_{0}$ and then study the global dynamics of system (5). Since the second equation of system (5) is decoupled from the other equations, it suffices to study the following system:

$$
\begin{aligned}
\frac{d S(t)}{d t}= & \Lambda(t)-f(t, S(t), I(t))-\mu(t) S(t)+\alpha(t) R(t), \\
\frac{d I(t)}{d t}= & \left(1-\tau^{\prime}(t)\right) e^{-\int_{t-\tau(t)}^{t} \mu(r) d r} f(t-\tau(t), S(t-\tau(t)), \\
& I(t-\tau(t)))-(\mu(t)+d(t)+\gamma(t)) I(t), \\
\frac{d R(t)}{d t}= & \gamma(t) I(t)-\mu(t) R(t)-\alpha(t) R(t) .
\end{aligned}
$$

It is easy to see that the scalar linear periodic equation

$$
\frac{d S(t)}{d t}=\Lambda(t)-\mu(t) S(t)
$$


has a unique positive $\omega$-periodic solution

$$
S^{*}(t)=\left[\int_{0}^{t} \Lambda(r) e^{\int_{0}^{r} \mu(s) d s} d r+\frac{\int_{0}^{\omega} \Lambda(r) e^{\int_{0}^{r} \mu(s) d s} d r}{e^{\int_{0}^{\omega} \mu(s) d s}-1}\right] e^{-\int_{0}^{t} \mu(s) d s},
$$

which is globally attractive in $\mathbb{R}$.

Linearizing system (7) at the disease-free periodic solution $\left(S^{*}(t), 0,0\right)$, we obtain the following periodic linear equation for the infective variable $I$ :

$$
\frac{d I(t)}{d t}=c(t) I(t-\tau(t))-b(t) I(t)
$$

where $c(t)=\left(1-\tau^{\prime}(t)\right) a(t) \frac{\partial f\left(t-\tau(t), S^{*}(t-\tau(t)), 0\right)}{\partial I}$ and $b(t)=\mu(t)+d(t)+\gamma(t)$.

Let $\hat{\tau}=\max _{0 \leq t \leq \omega} \tau(t), C=C([-\hat{\tau}, 0], \mathbb{R})$, and $C^{+}=C\left([-\hat{\tau}, 0], \mathbb{R}_{+}\right)$. Then $\left(C, C^{+}\right)$is an ordered Banach space equipped with the maximum norm and the positive cone $C^{+}$. For any given continuous function $v:[-\hat{\tau}, \sigma) \longrightarrow \mathbb{R}$ with $\sigma>0$, we define $v_{t} \in C$ by $v_{t}(\theta)=v(t+\theta), \forall \theta \in[-\hat{\tau}, 0]$, for any $t \in[0, \sigma)$. Let $F: \mathbb{R} \longrightarrow \mathscr{L}(C, \mathbb{R})$ be a map and $V(t)$ be a continuous function on $\mathbb{R}$ defined as follows:

$$
F(t) \phi=c(t) \phi(-\tau(t)), \quad V(t)=b(t) .
$$

Then the linear system (9) can be written as

$$
\frac{d v(t)}{d t}=F(t) v_{t}-V(t) v(t)
$$

Then the internal evolution of infective compartment $I$ is described by the following evolution system

$$
\frac{d v(t)}{d t}=-V(t) v(t)
$$

Let $\Phi(t, s), t \geq s$, be the evolution operator of the above linear system; that is, $\Phi(t)$ satisfies

$$
\frac{\partial}{\partial t} \Phi(t, s)=-V(t) \Phi(t, s), \quad \forall t \geq s, \quad \text { and } \quad \Phi(s, s)=I, \quad \forall s \in \mathbb{R}
$$

It then easily follows that

$$
\Phi(t, s)=e^{-\int_{s}^{t} b(r) d r}, \quad \forall t \geq s, s \in \mathbb{R}
$$

Let $C_{\omega}$ be the ordered Banach space of all continuous and $\omega$-periodic functions from $\mathbb{R}$ to $\mathbb{R}$, equipped with the maximum norm and the positive cone $C_{\omega}^{+}=\{v \in$ $\left.C_{\omega}: v(t) \geq 0, \forall t \in \mathbb{R}\right\}$. 
Suppose that $v \in C_{\omega}$ is the initial distribution of infectious individuals. Then for any given $s \geq 0, F(t-s) v_{t-s}$ is the distribution of newly infectious individuals at time $t-s$, which is produced by the infectious individuals who were introduced over the time interval $[t-s-\hat{\tau}, t-s]$. Then $\Phi(t, t-s) F(t-s) v_{t-s}$ is the distribution of those infectious individuals who newly became infectious at time $t-s$ and remain in the infectious compartments at time $t$. It follows that

$$
\int_{0}^{\infty} \Phi(t, t-s) F(t-s) v_{t-s} d s=\int_{0}^{\infty} \Phi(t, t-s) F(t-s) v(t-s+\cdot) d s
$$

is the distribution of accumulative new infectious at time $t$ produced by all those infectious individuals introduced at all previous time to $t$.

We define the next generation operator $L: C_{\omega} \longrightarrow C_{\omega}$ by

$$
[L v](t)=\int_{0}^{\infty} \Phi(t, t-s) F(t-s) v(t-s+\cdot) d s, \quad \forall t \in \mathbb{R}, v \in C_{\omega} .
$$

Following Zhao (2017a), we define $R_{0}=r(L)$, the spectral radius of $L$. For any given $t \geq 0$, let $\hat{P}(t)$ be the solution map of system (9); that is, $\hat{P}(t) \psi=u_{t}(\psi)$, where $u(t, \psi)$ is the unique solution of system (9) with $u_{0}=\psi \in C$. Then $\hat{P}:=\hat{P}(\omega)$ is the Poincaré (period) map associated with linear system (9). Let $r(\hat{P})$ be the spectral radius of $\hat{P}$. In view of Zhao (2017a, Theorem 2.1 ), we have the following result.

Lemma $1 R_{0}-1$ has the same sign as $r(\hat{P})-1$.

Let $P_{\lambda}$ be the Poincaré map on $C$ of the following linear periodic system with parameter $\lambda \in(0, \infty)$ :

$$
\frac{d u(t)}{d t}=\frac{1}{\lambda} F(t) u_{t}-V(t) u(t), \quad t \geq 0
$$

The following observation comes from Zhao (2017a, Theorem 2.2).

Lemma 2 If $R_{0}>0$, then $\lambda=R_{0}$ is the unique solution of $r\left(P_{\lambda}\right)=1$.

For any given $\lambda \in(0, \infty)$, we choose $v_{0} \in \operatorname{Int}\left(C^{+}\right)$and define

$$
a_{n}=\left\|P_{\lambda} v_{n-1}\right\|_{C}, \quad v_{n}=\frac{P_{\lambda} v_{n-1}}{a_{n}}, \quad \forall n \geq 1 .
$$

By Liang et al. (2017, Lemma 2.5), it follows that if $\lim _{n \rightarrow \infty} a_{n}$ exists, then $r\left(P_{\lambda}\right)=$ $\lim _{n \rightarrow \infty} a_{n}$. Thus, we can solve $r\left(P_{\lambda}\right)=1$ for $\lambda$ numerically via the bisection method.

Let $X=C\left([-\hat{\tau}, 0], \mathbb{R}_{+}^{3}\right)$. Then we have the following result for system (7).

Lemma 3 For any $\varphi \in X$, system (7) has a unique nonnegative solution $u(t, \varphi)$ with $u_{0}=\varphi$ for all $t \geq 0$, and solution are also ultimately bounded. 
Proof For any $\varphi=\left(\varphi_{1}, \varphi_{2}, \varphi_{3}\right) \in X$, we define

$$
\begin{aligned}
& \tilde{f}(t, \varphi) \\
& \quad=\left(\begin{array}{l}
\Lambda(t)-f\left(t, \varphi_{1}(0), \varphi_{2}(0)\right)-\mu(t) \varphi_{1}(0)+\alpha(t) \varphi_{3}(0) \\
\left(1-\tau^{\prime}(t)\right) e^{-\int_{t-\tau(t)}^{t} \mu(r) d r} f\left(t-\tau(t), \varphi_{1}(-\tau(t)), \varphi_{2}(-\tau(t))\right)-(\mu(t)+d(t)+\gamma(t)) \varphi_{2}(0) \\
\gamma(t) \varphi_{2}(0)-\mu(t) \varphi_{3}(0)-\alpha(t) \varphi_{3}(0)
\end{array}\right) .
\end{aligned}
$$

Note that $\tilde{f}(t, \varphi)$ is continuous in $(t, \varphi) \in \mathbb{R}_{+} \times X$ and $\tilde{f}(t, \varphi)$ is Lipschitz in $\varphi$ on each compact subset of $X$. By Hale and Verduyn Lunel (1993, Theorems 2.2.1 and 2.2.3), it then follows that system (7) has a unique solution $u(t, \varphi)$ on its maximal interval $\left[0, \sigma_{\varphi}\right)$ of existence with $u_{0}=\varphi$.

Let $\varphi=\left(\varphi_{1}, \varphi_{2}, \varphi_{3}\right) \in X$ be given. If $\varphi_{i}(0)=0$ for some $i \in\{1,2,3\}$, then $\tilde{f}_{i}(t, \varphi) \geq 0$. By Smith (1995, Theorem 5.2.1), it follows that for any $\varphi \in X$, the solution $u(t, \varphi)$ of system (7) with $u_{0}=\varphi$ is nonnegative for all $t \in\left[0, \sigma_{\varphi}\right)$. Define

$$
D:=\left\{\psi \in C\left([-\hat{\tau}, 0], \mathbb{R}_{+}^{4}\right): \psi_{2}(0)=\int_{-\tau(0)}^{0} e^{-\int_{s}^{0} \mu(r) d r} f\left(s, \psi_{1}(s), \psi_{3}(s)\right) d s\right\}
$$

It then easily follows that for any $\psi \in D$, system (5) has a unique nonnegative solution $v(t, \psi)=(S(t), E(t), I(t), R(t))$ satisfying $v_{0}=\psi$ for all $t \in\left[0, \sigma_{\varphi}\right)$.

Let $N(t)=S(t)+E(t)+I(t)+R(t)$. Then we have

$$
\frac{d N(t)}{d t}=\Lambda(t)-\mu(t) N(t)-d(t) I(t) \leq \Lambda(t)-\mu(t) N(t)
$$

for all $t \in\left[0, \sigma_{\varphi}\right)$. Thus, $\mathrm{S}(\mathrm{t}), E(t), \mathrm{I}(\mathrm{t})$ and $\mathrm{R}(\mathrm{t})$ are bounded on $t \in\left[0, \sigma_{\varphi}\right)$. Therefore, Hale and Verduyn Lunel (1993, Theorem 2.3.1) implies that $\sigma_{\varphi}=\infty$. It follows that

$$
\frac{d N(t)}{d t}=\Lambda(t)-\mu(t) N(t)-d(t) I(t) \leq \Lambda(t)-\mu(t) N(t), \quad t \geq 0
$$

Then the global stability of $S^{*}(t)$ for system (8), together with the comparison argument, implies that solutions of system (5) with initial data in $D$, and hence system (7) in $X$, exist globally on $[0, \infty)$ and are also ultimately bounded.

Let

$$
Y:=C\left([-\tau(0), 0], \mathbb{R}_{+}^{2}\right) \times \mathbb{R}_{+}
$$

Lemma 4 For any $\varphi \in Y$, system (7) has a unique nonnegative solution $u(t, \varphi)$ with $u_{0}=\varphi$ for all $t \geq 0$.

Proof Let $\bar{\tau}=\min _{t \in[0, \omega]} \tau(t)$. For any $t \in[0, \bar{\tau}]$, since $t-\tau(t)$ is strictly increasing in $t$, we have

$$
-\tau(0)=0-\tau(0) \leq t-\tau(t) \leq \bar{\tau}-\tau(\bar{\tau}) \leq \bar{\tau}-\bar{\tau}=0,
$$


and hence,

$$
S(t-\tau(t))=\varphi_{1}(t-\tau(t)) \text { and } I(t-\tau(t))=\varphi_{2}(t-\tau(t)) .
$$

Therefore, we have the following ordinary differential equations for $t \in[0, \bar{\tau}]$ :

$$
\begin{aligned}
\frac{d S(t)}{d t}= & \Lambda(t)-f(t, S(t), I(t))-\mu(t) S(t)+\alpha(t) R(t), \\
\frac{d I(t)}{d t}= & \left(1-\tau^{\prime}(t)\right) e^{-\int_{t-\tau(t)}^{t} \mu(r) d r} f\left(t-\tau(t), \varphi_{1}(t-\tau(t)),\right. \\
& \left.\varphi_{2}(t-\tau(t))\right)-(\mu(t)+d(t)+\gamma(t)) I(t), \\
\frac{d R(t)}{d t}= & \gamma(t) I(t)-\mu(t) R(t)-\alpha(t) R(t) .
\end{aligned}
$$

Given $\varphi \in Y$, the solution $(S(t), I(t), R(t))$ of the above system exists for $t \in[0, \bar{\tau}]$. In other words, we have obtained the values of $\psi_{1}(\theta)=S(\theta), \psi_{2}(\theta)=I(\theta)$ for $\theta \in[-\tau(0), \bar{\tau}]$ and $\psi_{3}(\theta)=R(\theta)$ for $\theta \in[0, \bar{\tau}]$.

For any $t \in[\bar{\tau}, 2 \bar{\tau}]$, we have

$$
-\tau(0)=0-\tau(0) \leq \bar{\tau}-\tau(\bar{\tau}) \leq t-\tau(t) \leq 2 \bar{\tau}-\tau(2 \bar{\tau}) \leq 2 \bar{\tau}-\bar{\tau}=\bar{\tau},
$$

and hence,

$$
S(t-\tau(t))=\psi_{1}(t-\tau(t)) \text { and } I(t-\tau(t))=\psi_{2}(t-\tau(t)) .
$$

Solving the following ordinary differential equations for $t \in[\bar{\tau}, 2 \bar{\tau}]$ with $S(\bar{\tau})=$ $\psi_{1}(\bar{\tau}), I(\bar{\tau})=\psi_{2}(\bar{\tau}), R(\bar{\tau})=\psi_{3}(\bar{\tau}):$

$$
\begin{aligned}
\frac{d S(t)}{d t}= & \Lambda(t)-f(t, S(t), I(t))-\mu(t) S(t)+\alpha(t) R(t), \\
\frac{d I(t)}{d t}= & \left(1-\tau^{\prime}(t)\right) e^{-\int_{t-\tau(t)}^{t} \mu(r) d r} f\left(t-\tau(t), \psi_{1}(t-\tau(t)),\right. \\
& \left.\psi_{2}(t-\tau(t))\right)-(\mu(t)+d(t)+\gamma(t)) I(t), \\
\frac{d R(t)}{d t}= & \gamma(t) I(t)-\mu(t) R(t)-\alpha(t) R(t) .
\end{aligned}
$$

We then get the solution $(S(t), I(t), R(t))$ on $[\bar{\tau}, 2 \bar{\tau}]$. Repeating this procedure for $t \in[2 \bar{\tau}, 3 \bar{\tau}],[3 \bar{\tau}, 4 \bar{\tau}], \ldots$, it then follows that for any $\varphi \in Y$, system (7) has a unique solution $u(t, \varphi)$ with $u_{0}=\varphi$ for all $t \geq 0$.

Remark 1 By the uniqueness of solutions in Lemmas 3 and 4, it follows that for any $\psi \in X$ and $\phi \in Y$ with $\psi_{1}(\theta)=\phi_{1}(\theta), \psi_{2}(\theta)=\phi_{2}(\theta)$, for all $\theta \in[-\tau(0), 0]$ and $\psi_{3}=\phi_{3}$, we have $w(t, \psi)=v(t, \phi)$ for all $t \geq 0$, where $w(t, \psi)$ and $v(t, \phi)$ are solutions of system (7) satisfying $w_{0}=\psi$ and $v_{0}=\phi$, respectively. 
Let

$$
W=C([-\tau(0), 0], \mathbb{R}), \quad W^{+}=C\left([-\tau(0), 0], \mathbb{R}_{+}\right) .
$$

For any given $t \geq 0$, let $P(t)$ be the solution map of the scalar linear periodic system (9) on $W$; that is, $P(t) \phi=v_{t}(\phi), t \geq 0$, where $v(t, \phi)$ is the unique solution of system (9) satisfying $v_{0}=\phi \in W$. By similar arguments to those in Lou and Zhao (2017, Lemma 3.5), we have the following result.

Lemma $5 P(t)$ is an $\omega$-periodic semiflow on $W$ in the sense that $(i) P(0)=I$; (ii) $P(t+\omega)=P(t) \circ P(\omega)$ for all $t \geq 0$; and (iii) $P(t) \phi$ is continuous in $(t, \phi) \in$ $[0, \infty) \times W$.

Let $P:=P(\omega)$ be the Poincaré map associated with system (9) on $W$ and $r(P)$ be its spectral radius. We first prove that the solution map $P(t)$ is monotone for each $t \geq 0$. For any given $\varphi, \psi \in W$ with $\varphi \geq \psi$. Let $\bar{v}(t)=v(t, \varphi)$ and $v(t)=v(t, \psi)$ be the unique solution of system (9) with $\bar{v}_{0}=\varphi$ and $v_{0}=\psi$, respectively. Since

$$
-\tau(0)=0-\tau(0) \leq t-\tau(t) \leq \bar{\tau}-\tau(\bar{\tau}) \leq \bar{\tau}-\bar{\tau}=0, \quad \forall t \in[0, \bar{\tau}],
$$

we have

$$
\bar{v}(t-\tau(t))=\varphi(t-\tau(t)) \text { and } v(t-\tau(t))=\psi(t-\tau(t)), \quad \forall t \in[0, \bar{\tau}] .
$$

Therefore, we have the following ordinary differential equations for $\forall t \in[0, \bar{\tau}]$,

$$
\begin{aligned}
& \frac{d I(t)}{d t}=c(t) \varphi(t-\tau(t))-b(t) I(t), \\
& \frac{d I(t)}{d t}=c(t) \psi(t-\tau(t))-b(t) I(t) .
\end{aligned}
$$

Given $\varphi, \psi \in W$, the solution $I(t)$ of the above equations exists for all $t \in[0, \bar{\tau}]$. In the view of $\bar{v}(0)=\varphi(0) \geq \psi(0)=v(0)$, the comparison theorem for cooperative ordinary differential systems implies that $\bar{v}(t) \geq v(t)$ for all $t \in[0, \bar{\tau}]$. Repeating this procedure for $t \in[\bar{\tau}, 2 \bar{\tau}],[2 \bar{\tau}, 3 \bar{\tau}], \ldots$, it follows that $v(t, \varphi) \geq v(t, \psi)$ for all $t \in[0, \infty)$. This implies that the solution map $P(t)$ is monotone for each $t \geq 0$. Now we show that the solution map $P(t)$ is eventually strongly monotone.

Lemma 6 For any $\varphi$ and $\psi$ in $W$ with $\varphi>\psi$ (that is, $\varphi \geq \psi$, but $\varphi \neq \psi$ ), the solutions $\bar{v}(t)$ and $v(t)$ of system (9) with $\bar{v}_{0}=\varphi$ and $v_{0}=\psi$, respectively, satisfy $\bar{v}(t)>v(t)$ for all $t \geq \hat{\tau}$, and hence, $P(t) \varphi \gg P(t) \psi$ in $W$ for all $t \geq \hat{\tau}+\tau(0)$. 
Proof We first show that $\bar{v}\left(t_{0}\right)>v\left(t_{0}\right)$ for some $t_{0} \in[0, \hat{\tau}]$. Assume, by contradiction, that $\bar{v}(t)=v(t)$ for all $t \in[0, \hat{\tau}]$. Then $\frac{d \bar{v}(t)}{d t}=\frac{d v(t)}{d t}$ for all $t \in(0, \hat{\tau})$, and hence,

$$
\begin{aligned}
\frac{d \bar{v}(t)}{d t}= & \left(1-\tau^{\prime}(t)\right) a(t) \frac{\partial f\left(t-\tau(t), S^{*}(t-\tau(t)), 0\right)}{\partial \bar{v}} \bar{v}(t-\tau(t)) \\
& -(\mu(t)+d(t)+\gamma(t)) \bar{v}(t), \\
\frac{d v(t)}{d t}= & \left(1-\tau^{\prime}(t)\right) a(t) \frac{\partial f\left(t-\tau(t), S^{*}(t-\tau(t)), 0\right)}{\partial v} v(t-\tau(t)) \\
& -(\mu(t)+d(t)+\gamma(t)) v(t),
\end{aligned}
$$

that is,

$$
\left(1-\tau^{\prime}(t)\right) a(t) \frac{\partial f\left(t-\tau(t), S^{*}(t-\tau(t)), 0\right)}{\partial \bar{v}}[\bar{v}(t-\tau(t))-v(t-\tau(t))]=0, \forall t \in[0, \hat{\tau}]
$$

It follows that $\bar{v}(t-\tau(t))=v(t-\tau(t))$ for all $t \in[0, \hat{\tau}]$. Since $-\tau(0)=0-\tau(0) \leq$ $t-\tau(t) \leq \hat{\tau}-\tau(\hat{\tau}), \forall t \in[0, \hat{\tau}]$, and $\hat{\tau}-\tau(\hat{\tau}) \geq 0$, we have $\varphi(\theta)=\psi(\theta)$ for all $\theta \in[-\tau(0), 0]$, which is a contradiction to the assumption $\varphi>\psi$ in $W$.

Let

$$
\begin{aligned}
g(t, \xi):= & \left(1-\tau^{\prime}(t)\right) a(t) \frac{\partial f\left(t-\tau(t), S^{*}(t-\tau(t)), 0\right)}{\partial v} \\
& \times v(t-\tau(t))-(\mu(t)+d(t)+\gamma(t)) \xi .
\end{aligned}
$$

By (A2), $\frac{\partial f(t, S, 0)}{\partial I}$ is non-decreasing for all $S>0$. It then follows that for all $t \geq t_{0}$,

$$
\begin{aligned}
\frac{d \bar{v}(t)}{d t}= & \left(1-\tau^{\prime}(t)\right) a(t) \frac{\partial f\left(t-\tau(t), S^{*}(t-\tau(t)), 0\right)}{\partial \bar{v}} \bar{v}(t-\tau(t)) \\
& -(\mu(t)+d(t)+\gamma(t)) \bar{v}(t) \\
\geq & \left(1-\tau^{\prime}(t)\right) a(t) \frac{\partial f\left(t-\tau(t), S^{*}(t-\tau(t)), 0\right)}{\partial v} v(t-\tau(t)) \\
& -(\mu(t)+d(t)+\gamma(t)) \bar{v}(t) \\
= & g(t, \bar{v}(t))
\end{aligned}
$$

and hence,

$$
\frac{d \bar{v}(t)}{d t}-g(t, \bar{v}(t)) \geq 0=\frac{d v(t)}{d t}-g(t, v(t)), \quad \forall t \geq t_{0} .
$$

Since $\bar{v}\left(t_{0}\right)>v\left(t_{0}\right)$, the comparison theorem for ordinary differential equations in Walter (1997, Theorem 4) implies that $\bar{v}(t)>v(t)$ for all $t \geq t_{0}$. Since $t_{0} \in[0, \hat{\tau}]$, $\bar{v}(t)>v(t)$ for all $t \geq \hat{\tau}$, and hence, $P(t) \varphi \gg P(t) \psi$ for all $t \geq \hat{\tau}+\tau(0)$.

Let $Q(t)$ be the solution maps of system (7) on $Y$; that is, $Q(t) \phi=v_{t}(\phi), t \geq 0$, where $v(t, \phi)$ is the unique solution of system (7) satisfying $v_{0}=\phi \in Y$. By the arguments similar to those in Lou and Zhao (2017, Lemma 3.5), we have the following result. 
Lemma $7 Q(t)$ is an $\omega$-periodic semiflow on $Y$ in the sense that (i) $Q(0)=I$; (ii) $Q(t+\omega)=Q(t) \circ Q(\omega)$ for all $t \geq 0$; and (iii) $Q(t) \phi$ is continuous in $(t, \phi) \in$ $[0, \infty) \times Y$.

We also need the following observation in our analysis of system (7).

Lemma 8 Assume that $f(t)$ is a nonnegative continuous and $\omega$-periodic function on $\mathbb{R}_{+}$with $\int_{0}^{\omega} f(t) d t>0$, and $g(t)$ is a continuous function on $\mathbb{R}_{+}$. If $\lim _{t \rightarrow \infty} g(t)$ $=0$, then any solution $u(t)$ of the linear non-homogeneous equation

$$
u^{\prime}(t)=-f(t) u(t)+g(t), t \geq 0
$$

satisfies $\lim _{t \rightarrow \infty} u(t)=0$.

Proof Let $U(t, s)=e^{-\int_{s}^{t} f(r) d r}$ and $M:=\frac{1}{\omega} \int_{0}^{\omega} f(t) d t$. For any $t \geq s \geq 0$, letting $n=\left[\frac{t-s}{\omega}\right]$, we then have

$$
\begin{aligned}
\int_{s}^{t} f(r) d r & =\int_{s}^{s+n \omega} f(r) d r+\int_{s+n \omega}^{t} f(r) d r \\
& =n \int_{0}^{\omega} f(r) d r+\int_{s+n \omega}^{t} f(r) d r \\
& =n \omega M+\int_{s}^{t-n \omega} f(r) d r .
\end{aligned}
$$

Let $K:=e^{M \omega}$. Since $M>0$ and $n \omega \leq t-s<(n+1) \omega$, it follows that

$$
U(t, s)=e^{-n \omega M} e^{-\int_{s}^{t-n \omega} f(r) d r} \leq e^{-(t-s-\omega) M}=K e^{-M(t-s)}, \quad \forall t \geq s \geq 0 .
$$

Let $\varepsilon>0$ be given. Since $\lim _{t \rightarrow \infty} g(t)=0$, there exists a sufficiently large $t_{0}>0$ such that $|g(t)|<\varepsilon, \forall t \geq t_{0}$. It then follows that the solution $u(t)$ of (11) satisfies

$$
u(t)=U\left(t, t_{0}\right) u\left(t_{0}\right)+\int_{t_{0}}^{t} U(t, s) g(s) d s, \quad \forall t \geq t_{0},
$$

and hence,

$$
\begin{aligned}
|u(t)| & \leq K e^{-M\left(t-t_{0}\right)}\left|u\left(t_{0}\right)\right|+\varepsilon \int_{t_{0}}^{t} K e^{-M(t-s)} d s \\
& \leq K e^{-M\left(t-t_{0}\right)}\left|u\left(t_{0}\right)\right|+\frac{\varepsilon K}{M}\left(1-e^{-M\left(t-t_{0}\right)}\right), \forall t \geq t_{0} .
\end{aligned}
$$

This implies that $\lim _{t \rightarrow \infty} u(t)=0$.

Now we are ready to prove the main result of this section.

Theorem 1 Let (A1) and (A2) hold. Then the following statements are valid: 
(i) If $r(P)<1$, then the disease-free periodic solution $\left(S^{*}(t), 0,0\right)$ is globally attractive for system (7) in $Y$.

(ii) If $r(P)>1$, then system (7) admits a positive $\omega$-periodic solution $(\bar{S}(t), \bar{I}(t)$, $\bar{R}(t))$ and these exists a real number $\eta>0$ such that the solution $v(t, \phi)=$ $(S(t), I(t), R(t))$ satisfies $\liminf _{t \rightarrow \infty} I(t) \geq \eta$ for any $\phi \in Y$ with $\phi_{2}(0)>0$.

Proof Our proof is motivated by the arguments in Zhao (2017a, Theorem 3.1). By Hale and Verduyn Lunel (1993, Theorem 3.6.1), it follows that for each $t \geq \hat{\tau}$, the linear operator $\hat{P}(t)$ is compact on $C$, and hence $P(t)$ is also compact on $W$ for any $t \geq \hat{\tau}$. In view of Lemma $6, P(t)$ is strongly positive on $W$ for any $t \geq \hat{\tau}+\tau(0)$. Thus, for any $t \geq \tilde{\tau}=\hat{\tau}+\tau(0), P(t)$ is compact and strongly positive on $W$. Choose an integer $n_{0}>0$ such that $n_{0} \omega \geq \tilde{\tau}$. Since $P^{n_{0}}=P\left(n_{0} \omega\right)$, Liang and Zhao (2007, Lemma 3.1) implies that $r(P)$ is a simple eigenvalue of $P$ having a strongly positive eigenvector, and the modulus of any other eigenvalue is less than $r(P)$. Let $\mu=\frac{\ln r(P)}{\omega}$. By Wang and Zhao (2017a, Lemma 1), it then follows that there is a positive $\omega$-periodic function $v(t)$ such that $u(t)=e^{\mu t} v(t)$ is a positive solution of linear equation (9).

In the case where $r(P)<1$, let $P_{\epsilon}(t)$ be the solution maps of the following perturbed linear periodic equation on $W$ :

$$
\begin{aligned}
\frac{d I(t)}{d t}= & \left(1-\tau^{\prime}(t)\right) a(t) \frac{\partial f\left(t-\tau(t), S^{*}(t-\tau(t))+\epsilon, 0\right)}{\partial I} I(t-\tau(t))-(\mu(t) \\
& +d(t)+\gamma(t)) I(t),
\end{aligned}
$$

and $P_{\epsilon}:=P_{\epsilon}(\omega)$. Since $\lim _{\epsilon \rightarrow 0} r\left(P_{\epsilon}\right)=r(P)<1$, we can fix a sufficiently small number $\epsilon>0$ such that $r\left(P_{\epsilon}\right)<1$. It is easy to verify that $P_{\epsilon}(t)$ is also compact and strongly monotone on $W$ for each $t \geq \tilde{\tau}$. As discussed above, there is a positive $\omega$-periodic function $v_{\epsilon}(t)$ such that $u_{\epsilon}(t)=e^{\mu_{\epsilon} t} v_{\epsilon}(t)$ is a positive solution of system (12), where $\mu_{\epsilon}=\frac{\ln r\left(P_{\epsilon}\right)}{\omega}<0$. Clearly, $\lim _{t \rightarrow \infty} u_{\epsilon}(t)=0$.

In view of (10) and the global stability of $S^{*}(t)$ for system (8), there exists a sufficiently large integer $N_{1}>0$ such that $N_{1} \omega \geq \hat{\tau}$ and $S(t) \leq S^{*}(t)+\epsilon, \forall t \geq$ $N_{1} \omega-\hat{\tau}$. By assumption (A2), we then have

$$
\begin{aligned}
\frac{d I(t)}{d t} \leq & \left(\left(1-\tau^{\prime}(t)\right) a(t) \frac{\partial f\left(t-\tau(t), S^{*}(t-\tau(t))+\epsilon, 0\right)}{\partial I} I(t-\tau(t))\right) \\
& -(\mu(t)+d(t)+\gamma(t)) I(t),
\end{aligned}
$$

for all $t \geq N_{1} \omega$. Choose a sufficiently large number $k>0$ such that $I(t) \leq k u_{\epsilon}(t)$, $\forall t \in\left[N_{1} \omega, N_{1} \omega+\hat{\tau}\right]$. Thus, the comparison theorem for delay differential equations (see Smith 1995, Theorem 5.1.1) implies that $I(t) \leq k u_{\epsilon}(t), \forall t \geq N_{1} \omega+\hat{\tau}$. Thus, $\lim _{t \rightarrow \infty} I(t)=0$. By Lemma 8 with $f(t)=\mu(t)+\alpha(t)$ and $g(t)=\gamma(t) I(t)$, as applied to the third equation of system (7), it then follows that $\lim _{t \rightarrow \infty} R(t)=0$. Let $w(t):=S(t)-S^{*}(t)$. In view of (7) and (8), we have

$$
w^{\prime}(t)=-\mu(t) w(t)+(\alpha(t) R(t)-f(t, S(t), I(t)))
$$


Since $\lim _{t \rightarrow \infty} I(t)=0$ and $\lim _{t \rightarrow \infty} R(t)=0$, it follows that

$$
\lim _{t \rightarrow \infty}(\alpha(t) R(t)-f(t, S(t), I(t)))=0
$$

Now Lemma 8 implies that $\lim _{t \rightarrow \infty} w(t)=0$; that is, $\lim _{t \rightarrow \infty}\left(S(t)-S^{*}(t)\right)=0$. This proves statement (i).

In the case where $r(P)>1$, we will apply the persistence theory for periodic semiflows. Let $Y_{0}=\left\{\phi=\left(\phi_{1}, \phi_{2}, \phi_{3}\right) \in Y: \phi_{2}(0)>0\right\}$ and $\partial Y_{0}:=Y \backslash Y_{0}=\{\phi=$ $\left.\left(\phi_{1}, \phi_{2}, \phi_{3}\right) \in Y: \phi_{2}(0)=0\right\}$. Let $Q(t) \phi=v_{t}(\phi), \forall \phi \in Y$. Then $Q:=Q(\omega)$ is the Poincaré map associated with system (7) on $Y$ and $Q^{n}=Q(n \omega), \forall n \geq 0$.

From the second equation of system (7), it is easy to see that $Q(t) Y_{0} \subseteq Y_{0}$ for all $t \geq 0$. By Lemma 3 and Remark 1, the discrete-time system $\left\{Q^{n}: Y \rightarrow Y\right\}_{n \geq 0}$ is point dissipative. By Hale and Verduyn Lunel (1993, Theorem 3.6.1) and Remark 1, for each $t \geq \hat{\tau}, Q(t)$ is compact, and hence $Q^{n}$ is compact for sufficiently large $n$. It then follows from Zhao (2017b, Theorem 1.1.3) that $Q$ admits a strong global attractor in $Y$. Now we prove that $Q$ is uniformly persistent with respect to $\left(Y_{0}, \partial Y_{0}\right)$.

Let $M_{\delta}$ be the Poincaré map of the following perturbed linear periodic equation:

$$
\begin{aligned}
\frac{d I(t)}{d t}= & \left(1-\tau^{\prime}(t)\right) a(t)\left(\frac{\partial f\left(t-\tau(t), S^{*}(t-\tau(t)), 0\right)}{\partial I}-\delta\right) I(t-\tau(t))-(\mu(t) \\
& +d(t)+\gamma(t)) I(t) .
\end{aligned}
$$

Since $\lim _{\delta \rightarrow 0} r\left(M_{\delta}\right)=r(P)>1$, we can fix a sufficiently small $\delta$ such that $r\left(M_{\delta}\right)>$ 1. It follows that there is a small number $\eta_{0}>0$ such that

$$
f\left(t-\tau(t), S^{*}(t-\tau(t))-\eta_{0}, I\right) \geq\left(\frac{\partial f\left(t-\tau(t), S^{*}(t-\tau(t)), 0\right)}{\partial I}-\delta\right) I, \forall I \in\left[0, \eta_{0}\right] .
$$

Let $M_{1}=\left(S_{0}^{*}, 0,0\right)$, where $S_{0}^{*}(\theta)=S^{*}(\theta)$ for all $\theta \in[-\tau(0), 0]$. Then $Q(t) M_{1}=$ $\left(S_{t}^{*}, 0,0\right), \forall t \geq 0$, and $Q\left(M_{1}\right)=M_{1}$. Since $\lim _{\phi \rightarrow M_{1}}\left\|Q(t) \phi-Q(t) M_{1}\right\|=0$ uniformly for $t \in[0, \omega]$, there exists $\eta_{1}=\eta_{1}\left(\eta_{0}\right)>0$ such that for any $\phi \in Y_{0}$ with $\left\|\phi-M_{1}\right\|<\eta_{1}$, we have $\left\|Q(t) \phi-Q(t) M_{1}\right\|<\eta_{0}$ for all $t \in[0, \omega]$. We further have the following claim.

Claim $\lim \sup _{n \rightarrow \infty}\left\|Q^{n}(\phi)-M_{1}\right\| \geq \eta_{1} \quad$ for all $\phi \in Y_{0}$.

Suppose, by contradiction, that $\lim _{\sup _{n \rightarrow \infty}}\left\|Q^{n}(\psi)-M_{1}\right\|<\eta_{1}$ for some $\psi \in Y_{0}$. Then there exists an integer $N_{2} \geq 1$ such that $\left\|Q^{n}(\psi)-M_{1}\right\|<\eta_{1}$ for all $n \geq N_{2}$. For any $t \geq N_{2} \omega$, we have $t=n \omega+t^{\prime}$ with $n \geq N_{2}$ and $t^{\prime} \in[0, \omega]$, and hence,

$$
\left\|Q(t) \psi-Q(t) M_{1}\right\|=\left\|Q\left(t^{\prime}\right)\left(Q^{n}(\psi)\right)-Q\left(t^{\prime}\right) M_{1}\right\|<\eta_{0}, \forall t \geq N_{2} \omega .
$$

It follows that $S(t-\tau(t))>S^{*}(t-\tau(t))-\eta_{0}$ for all $t \geq N_{2} \omega+\hat{\tau}$. In view of (A2), we see that $f(t, S, I)$ is nondecreasing in $S$. Thus, $v(t, \psi)=(S(t), I(t), R(t))$ satisfies 


$$
\begin{aligned}
\frac{d I(t)}{d t}= & \left(1-\tau^{\prime}(t)\right) a(t) f(t-\tau(t), S(t-\tau(t)), \\
& I(t-\tau(t)))-(\mu(t)+d(t)+\gamma(t)) I(t) \\
\geq & \left(1-\tau^{\prime}(t)\right) a(t) f\left(t-\tau(t), S^{*}(t-\tau(t))-\eta_{0},\right. \\
& I(t-\tau(t)))-(\mu(t)+d(t)+\gamma(t)) I(t) \\
\geq & \left(1-\tau^{\prime}(t)\right) a(t)\left(\frac{\partial f\left(t-\tau(t), S^{*}(t-\tau(t)), 0\right)}{\partial I}-\delta\right) I(t-\tau(t)) \\
& -(\mu(t)+d(t)+\gamma(t)) I(t),
\end{aligned}
$$

for all $t \geq N_{2} \omega+\hat{\tau}$. Note that $r\left(M_{\delta}\right)>1$. As discussed earlier, there is a positive $\omega$-periodic function $v_{\delta}(t)$ such that $u_{\delta}=e^{\mu_{\delta} t} v_{\delta}(t)$ is a positive solution of system (13), where $\mu_{\delta}=\frac{\ln r\left(M_{\delta}\right)}{\omega}>0$. Since $Q(t) Y_{0} \subseteq Y_{0}, I(t)>0$ for all $t \geq 0$. We can choose a sufficiently small $k>0$ such that $I(t) \geq k u_{\delta}(t), \forall t \in\left[N_{2} \omega+\hat{\tau}, N_{2} \omega+2 \hat{\tau}\right]$. By the comparison theorem for delay differential equations (see Smith 1995, Theorem 5.1.1), it follows that $I(t) \geq k u_{\delta}(t), \forall t \geq N_{2} \omega+2 \hat{\tau}$. Clearly, $\lim _{t \rightarrow \infty} u_{\delta}(t)=\infty$. Thus, $\lim _{t \rightarrow \infty} I(t)=\infty$, which is a contradiction.

The above claim implies that $M_{1}$ is an isolated invariant set for $Q$ in $Y$ and $W^{S}\left(M_{1}\right) \bigcap Y_{0}=\emptyset$, where $W^{S}\left(M_{1}\right)$ is the stable set of $M_{1}$ for $Q$. Define

$$
M_{\partial}:=\left\{\phi \in \partial Y_{0}: Q^{n}(\phi) \in \partial Y_{0}, \forall n \geq 0\right\} .
$$

Since

$$
\frac{d I(t)}{d t} \geq-(\mu(t)+d(t)+\gamma(t)) I(t), \forall t \geq 0,
$$

it is easy to see that if $I\left(t_{0}\right)>0$ for some $t_{0} \geq 0$, then $I(t)>0$ for all $t \geq t_{0}$. This implies that $I(t)=0, \forall t \geq 0$, whenever $\phi \in M_{\partial}$. It then follows that $\omega(\phi)=M_{1}$ for any $\phi \in M_{\partial}$, and $M_{1}$ cannot form a cycle for $Q$ in $\partial Y_{0}$. By the acyclicity theorem on uniform persistence for maps (see Zhao 2017b, Theorem 1.3.1 and Remark 1.3.1), $Q: Y \rightarrow Y$ is uniformly persistent with respect to $\left(Y_{0}, \partial Y_{0}\right)$.

Define

$$
\begin{aligned}
& X_{0}=\left\{\psi=\left(\psi_{1}, \psi_{2}, \psi_{3}\right) \in X: \psi_{2}(0)>0\right\} \\
& \partial X_{0}:=X \backslash X_{0}=\left\{\psi=\left(\psi_{1}, \psi_{2}, \psi_{3}\right) \in X: \psi_{2}(0)=0\right\}
\end{aligned}
$$

Let $\hat{Q}(t)$ be the solution maps of system (7) on $X$; that is, $\hat{Q}(t) \psi=w_{t}(\psi), \forall t \geq 0$, where $w(t, \psi)$ is the unique solution of system (7) satisfying $w_{0}=\psi \in X$. Then $\hat{Q}:=\hat{Q}(\omega)$ is the Poincaré map associated with system (7) and $\hat{Q}^{n}:=\hat{Q}(n \omega)$, $\forall n \geq 0$.

From Hale and Verduyn Lunel (1993, Theorem 3.6.1), we know that $\hat{Q}(t)$ is compact on $X$ for each $t \geq \hat{\tau}$, and hence $\hat{Q}^{n}$ is compact for sufficiently large $n$. By Lemma 3 , solutions $w(t, \psi)$ are ultimately bounded on $[0, \infty)$, and hence, the discrete-time system $\left\{\hat{Q}^{n}: X \rightarrow X\right\}_{n \geq 0}$ is point dissipative. By Remark 1, it follows that $\hat{Q}$ is uniformly persistent with respect to $\left(X_{0}, \partial X_{0}\right)$. 
Note that for any integer $\hat{n}_{0}$ with $\hat{n}_{0} \omega \geq \tau, \hat{Q}^{\hat{n}_{0}}=\hat{Q}\left(\hat{n}_{0} \omega\right): X \rightarrow X$ is compact. By Zhao (2017b, Theorem 3.5.1), $\hat{Q}(t)$ is an $\alpha$-contraction with respect to an equivalent norm in $C\left([-\hat{\tau}, 0], \mathbb{R}^{3}\right)$ for any $t>0$. It then follows from Zhao (2017b, Theorem 1.3.10) that there exists a global attractor $A$ for $\hat{Q}: X_{0} \rightarrow X_{0}$ and $\hat{Q}$ has a fixed point $\psi^{*} \in A$, and hence, $w\left(t, \psi^{*}\right)=(\bar{S}(t), \bar{I}(t), \bar{R}(t))$ is an $\omega$-periodic solution of system (7) with $\psi^{*} \in X_{0}$. By Remark $1, v\left(t, \phi^{*}\right)=(\bar{S}(t), \bar{I}(t), \bar{R}(t))$ is also an $\omega$-periodic solution of system (7) with $\phi^{*} \in Y_{0}$, where $\phi_{1}^{*}(\theta)=\psi_{1}^{*}(\theta), \phi_{2}^{*}(\theta)=\psi_{2}^{*}(\theta)$, for all $\theta \in[-\tau(0), 0]$ and $\phi_{3}^{*}=\psi_{3}^{*}$. Then $\bar{S}(t) \geq 0, \bar{I}(t)>0$ and $\bar{R}(t) \geq 0$.

We claim that there exists some $\bar{t} \in[0, \omega]$ such that $\bar{S}(\bar{t})>0$. If not, then $\bar{S}(t) \equiv 0$ for all $t \geq 0$, due to the periodicity of $\bar{S}(t)$. Since $\Lambda(t)>0$ and $f(t, 0, I) \equiv 0$, we see from the first equation of system (7) that $0=\Lambda(t)+\alpha(t) \bar{R}(t)>0$, which is a contradiction. Since $\bar{S}(\bar{t})>0$ for some $\bar{t} \in[0, \omega]$, and

$$
\begin{aligned}
\left.\frac{d \bar{S}(t)}{d t}\right|_{\bar{S}(t)=0} & =\Lambda(t)-f(t, \bar{S}(t), \bar{I}(t))-\mu(t) \bar{S}(t)+\alpha(t) \bar{R}(t) \\
& =\Lambda(t)+\alpha(t) \bar{R}(t)>0, \forall t \geq \bar{t}
\end{aligned}
$$

it follows that $\bar{S}(t)>0$ for all $t \geq \bar{t}$. Now the periodicity of $\bar{S}(t)$ implies that $\bar{S}(t)>0$ for all $t \geq 0$. We claim that there exists some $\hat{t} \in[0, \omega]$ such that $\bar{R}(\hat{t})>0$. If not, then $\bar{R}(t) \equiv 0$ for all $t \geq 0$, due to the periodicity of $\bar{R}(t)$. Since $\gamma(t) \geq 0$ and $\int_{0}^{\omega} \gamma(t) d t>$ $0, \gamma(t) \not \equiv 0$, the third equation of system (7) implies that $0=\gamma(t) I(t) \not \equiv 0$ for all $t \geq 0$, which is a contradiction. Since $\bar{R}(\hat{t})>0$ for some $\hat{t} \in[0, \omega]$ and

$$
\frac{d \bar{R}(t)}{d t} \geq-(\mu(t)+\alpha(t)) \bar{R}(t)
$$

it follows that $\bar{R}(t)>0$ for all $t \geq \hat{t}$. Now the periodicity of $\bar{R}(t)$ implies that $\bar{R}(t)>0$ for all $t \geq 0$. Therefore, $v\left(t, \phi^{*}\right)=(\bar{S}(t), \bar{I}(t), \bar{R}(t))$ is a positive $\omega$-periodic solution of system (7) with $\phi^{*} \in Y_{0}$.

By Zhao (2017b, Theorem 1.3.6), it then follows that $Q: Y_{0} \rightarrow Y_{0}$ has a global attractor $A_{0}$. Since $A_{0}=Q\left(A_{0}\right)=Q(\omega) A_{0}$, we have $\phi_{2}(0)>0, \forall \phi \in A_{0}$. Let $B_{0}:=\bigcup_{t \in[0, \omega]} Q(t) A_{0}$. Then $\psi_{2}(0)>0$ for all $\psi \in B_{0}$. Moreover, $B_{0} \subseteq Y_{0}$, and Zhao (2017b, Theorem 3.1.1) implies that $\lim _{t \rightarrow \infty} d\left(Q(t) \phi, B_{0}\right)=0$ for all $\phi \in Y_{0}$. Define a continuous function $p: Y \rightarrow \mathbb{R}_{+}$by

$$
p(\phi)=\phi_{2}(0), \forall \phi \in Y
$$

Since $B_{0}$ is a compact subset of $Y_{0}$, we have $\inf _{\phi \in B_{0}} p(\phi)=\min _{\phi \in B_{0}} p(\phi)>0$. Consequently, there exists $\eta>0$ such that

$$
\liminf _{t \rightarrow \infty} I(t, \phi)=\liminf _{t \rightarrow \infty} p(Q(t) \phi) \geq \eta, \forall \phi \in Y_{0}
$$

This completes the proof. 
By the same arguments as in Lou and Zhao (2017, Lemma 3.8), we have $r(P)=$ $r(\hat{P})$. Combining Lemma 1 and Theorem 1, we have the following result on the global dynamics of system (7).

Theorem 2 Let (A1) and (A2) hold. Then the following statements are valid for system (7):

(i) If $R_{0}<1$, then the disease-free periodic solution $\left(S^{*}(t), 0,0\right)$ is globally attractive for system (7) in $Y$.

(ii) If $R_{0}>1$, then system (7) admits a positive $\omega$-periodic solution $(\bar{S}(t), \bar{I}(t), \bar{R}(t))$ and these exists a real number $\eta>0$ such that $v(t, \phi)=(S(t), I(t), R(t))$ satisfies $\liminf _{t \rightarrow \infty} I(t) \geq \eta$ for any $\phi \in Y$ with $\phi_{2}(0)>0$.

In the rest of this section, we derive the asymptotic behavior of the variable $E(t)$ in system (5). We have known that

$$
E(t)=\int_{t-\tau(t)}^{t} e^{-\int_{s}^{t} \mu(r) d r} f(s, S(s), I(s)) d s .
$$

In the case where $R_{0}<1$, we have

$$
\lim _{t \rightarrow \infty}\left[(S(t), I(t), R(t))-\left(S^{*}(t), 0,0\right)\right]=0 .
$$

It then follows from equation (14) that $\lim _{t \rightarrow \infty} E(t)=0$.

In the case where $R_{0}>1$, we have known that system (7) admits a positive $\omega$ periodic solution $(\bar{S}(t), \bar{I}(t), \bar{R}(t))$. By using the integral form (14), we obtain

$$
\bar{E}=\int_{t-\tau(t)}^{t} e^{-\int_{s}^{t} \mu(r) d r} f(s, \bar{S}(s), \bar{I}(s)) d s
$$

is also a positive $\omega$-periodic function. Consequently, we have the following result on the global dynamics of system (5).

Theorem 3 Let (A1) and (A2) hold. Then the following statements are valid for system (5):

(i) If $R_{0}<1$, then the disease-free periodic solution $\left(S^{*}(t), 0,0,0\right)$ is globally attractive for system (5) in $D$.

(ii) If $R_{0}>1$, then system (5) admits a positive $\omega$-periodic solution $(\bar{S}(t), \bar{E}(t), \bar{I}(t)$, $\bar{R}(t))$ and the se exists a real number $\eta>0$ such that the solution $(S(t), E(t), I(t)$, $R(t))$ of system (5) with for any $\phi \in D$ with $\phi_{3}(0)>0$ satisfies $\lim _{i n f} \operatorname{int}_{t \rightarrow \infty} I(t) \geq$ $\eta$.

\section{Numerical simulations}

To illustrate our results, in this section we apply our results to a special case and reveal the influence of the periodic time delay. 
Let us choose $f(t, S, I)=\frac{\beta(t) S I}{1+\varepsilon(t) I}$ and $\omega=365$ days. Based on the data of Mateus and Silva (2017), we take the following parameter values,

$$
\begin{aligned}
& \Lambda(t)=1000 / 25550(1+0.2 \cos (2 \pi t / 365)) \mathrm{day}^{-1}, \\
& \mu(t)=1 / 25550(1+0.2 \cos (2 \pi t / 365)) \mathrm{day}^{-1}, \\
& \alpha(t)=1 / 7(1+0.5 \cos (2 \pi t / 365)) \mathrm{day}^{-1}, \\
& \gamma(t)=1 / 2.2(1+0.1 \cos (2 \pi t / 365)) \mathrm{day}^{-1} .
\end{aligned}
$$

Any periodic function can be expressed as a sum of harmonic terms. For the sake of convenience, we assume that the periodic time delay is $\tau(t)=23(1+0.8 \cos (2 \pi t / 365))$ day, the disease-induced death rate is $d(t)=0.00079(1+0.2 \cos (2 \pi t / 365))$ day $^{-1}$, the transmission coefficient is $\beta(t)=0.0015(1+0.2 \cos (2 \pi t / 365)) \mathrm{day}^{-1}$, and $\varepsilon(t)=0.011$. It should be pointed out these parameters are chosen for illustrative purpose only, and may not be meaningful biologically.

Firstly, to computer the basic reproduction ratio $R_{0}$ numerically, we apply Lemma 2 and Liang et al. (2017, Lemma 2.5). With the above set of parameters, we obtain $R_{0}=3.1263>1$. When the initial functions are chosen as $S(\theta)=550, I(\theta)=1$, $R(0)=50$ for all $\theta \in[-\hat{\tau}, 0]$, we calculate $E(0) \approx 39.9441$. In this case, all compartments fluctuates periodically, which implies the disease will persist and exhibit periodic fluctuations eventually. Figure 1 illustrates the result and is coincident with Theorem 3 (ii). In order to understand the seasonal patterns of disease risk, we only consider the number of the infectious population in one year. Figure 2 shows that the number of infectious individuals is expected to peak between 840th day (April) and 910th day (June). Hence control strategies should aim to lower the number of infections during the course of a pandemic and postpone the timing of the peaks so that people have enough time to take appropriate measures. If we decrease the transmission coefficient to $0.2 \beta(t)$, and the initial functions are chosen as $S(\theta)=550, I(\theta)=73$, $R(0)=50$ for all $\theta \in[-\hat{\tau}, 0]$, and hence $E(0) \approx 327.0103$, then we calculate $R_{0}=0.6253<1$. In this case, from Theorem 3 (i), the susceptible population exhibits periodic fluctuations (constant as a special case), and the exposed, infectious and recovered populations all converge to zero, which means that the disease will be eliminated. Figure 3 illustrates the results above.

Secondly, we explore the influence of the transmission coefficient on $R_{0}$. Let $\beta(t)=$ $a(1+b \cos (2 \pi t / 365)), 0 \leq b \leq 1$, where $a$ is the mean contact rate, $b$ is the amplitude of fluctuations (or the strength of seasonal forcing). Fixed $b=0.2, R_{0}$ is strictly increasing with respect to the mean contact $a$ from Fig. 4. If fixed $a=0.0015$, then $R_{0}$ is decreasing with respect to the amplitude of fluctuations $b$ from Fig. 5. That is, the basic reproduction ratio $R_{0}$ depends not only on the mean contact rate, but also on the amplitude of fluctuations. Then we see that $R_{0}$ is highly sensitive to $\beta(t)$. This also shows that the transmission rate has an important role in the spread of the disease. Clearly, by taking some control measures such as isolation or vaccination effort, we assume that the control effort is $k(0 \leq k \leq 1)$; that is, the transmission rate becomes $(1-k) \beta(t)$. If we can decrease the transmission coefficient to $0.31 \beta(t)$, then $R_{0}<1$, which implies that the disease will die out, see Fig. 6. 


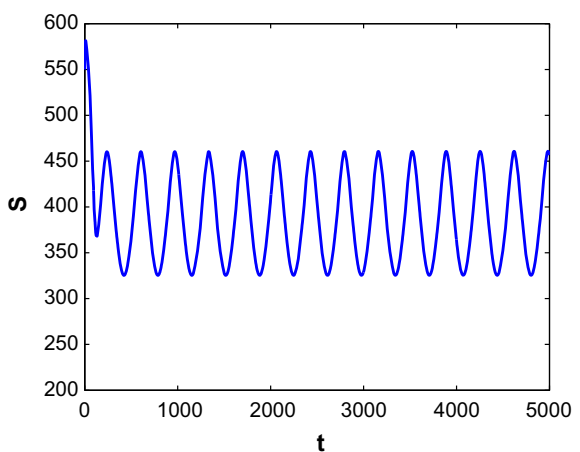

(a)

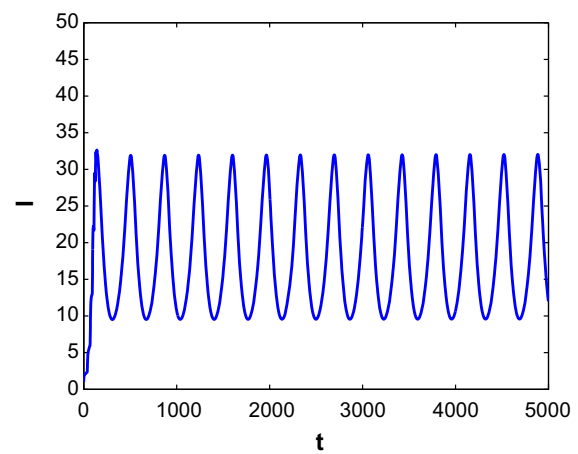

(c)

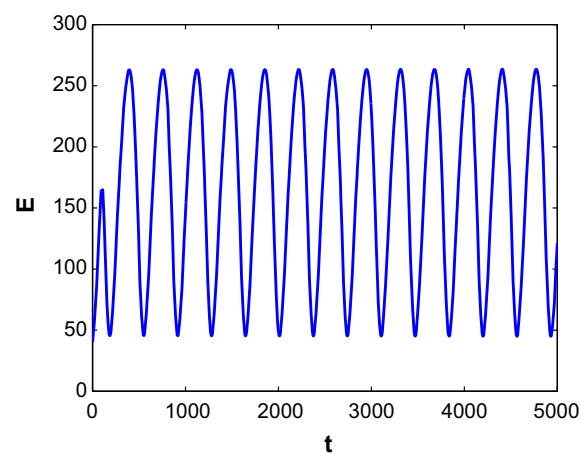

(b)

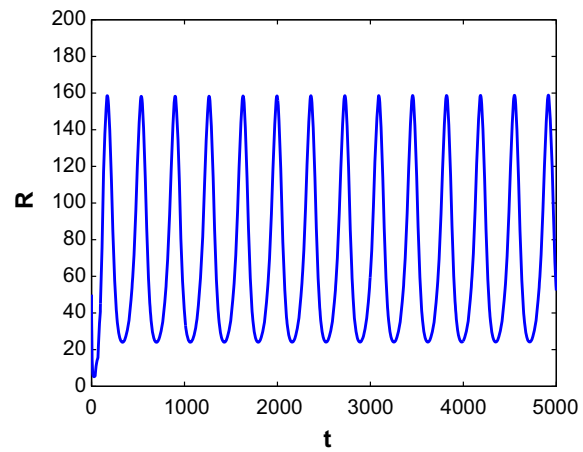

(d)

Fig. 1 Long-term behavior of the solution of system (5) when $R_{0}=3.1263>1$

Fig. 2 The curve of the number of the infectious population of system (5) when

$R_{0}=3.1263>1$ in one year

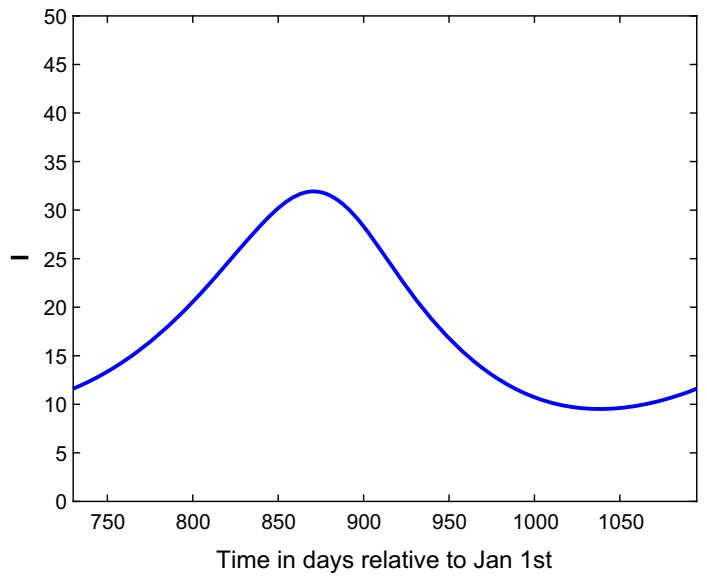




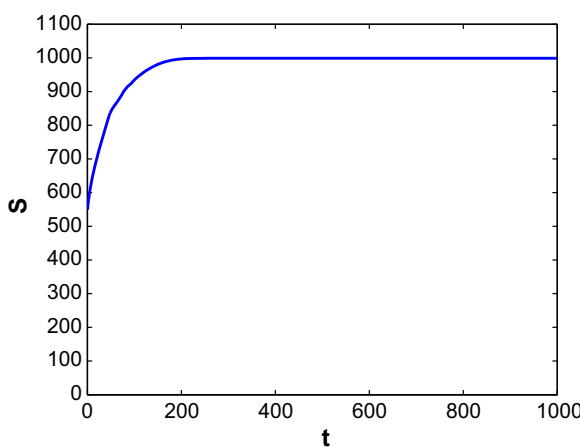

(a)

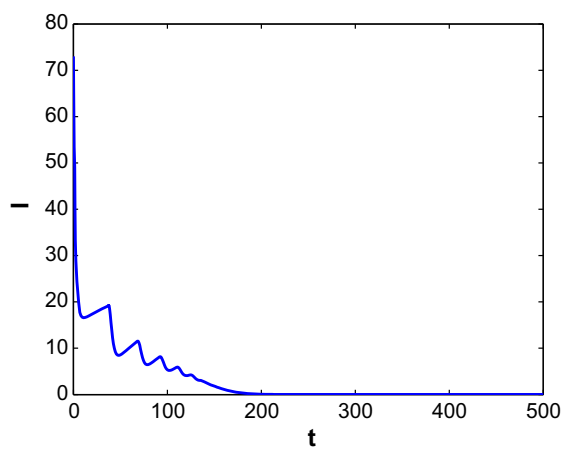

(c)

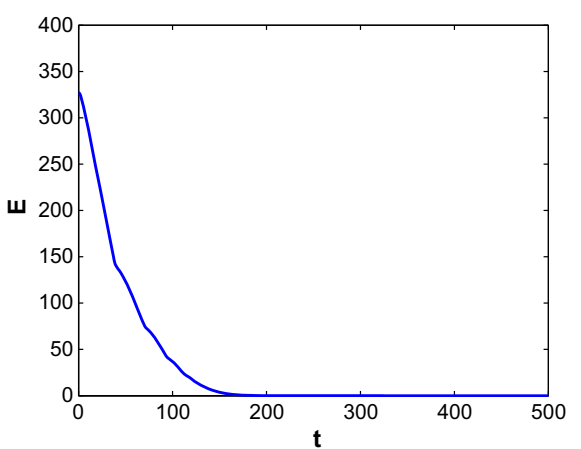

(b)

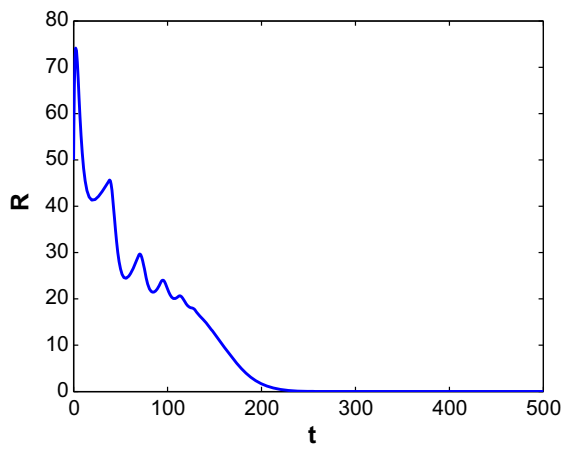

(d)

Fig. 3 Long-term behavior of the solution of system (5) when $R_{0}=0.6253<1$

Fig. 4 The basic reproduction ratio $R_{0}$ as a function of $a$ for system (5) fixed $b=0.2$

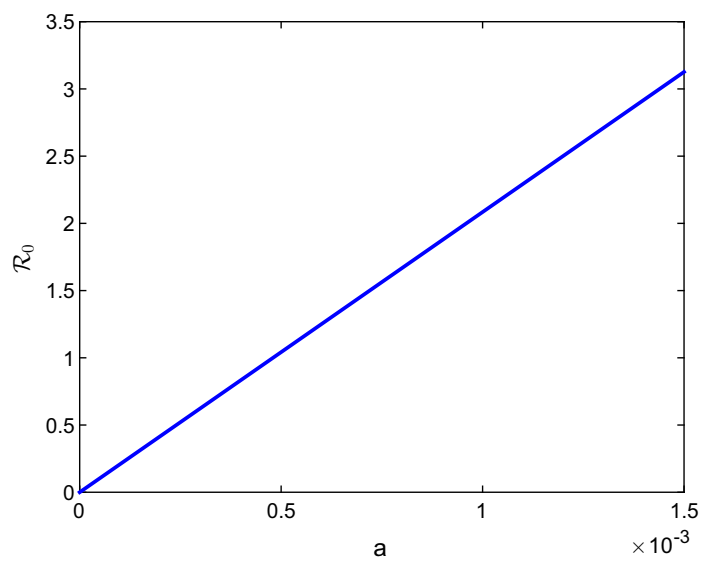


Fig. 5 The basic reproduction ratio $R_{0}$ as a function of $b$ for system (5) fixed $a=0.0015$

Fig. 6 The basic reproduction ratio $R_{0}$ as a function of $k$ for system (5)

Fig. 7 Comparison of the long-term behavior of the infectious population of system (5) with different latent periods
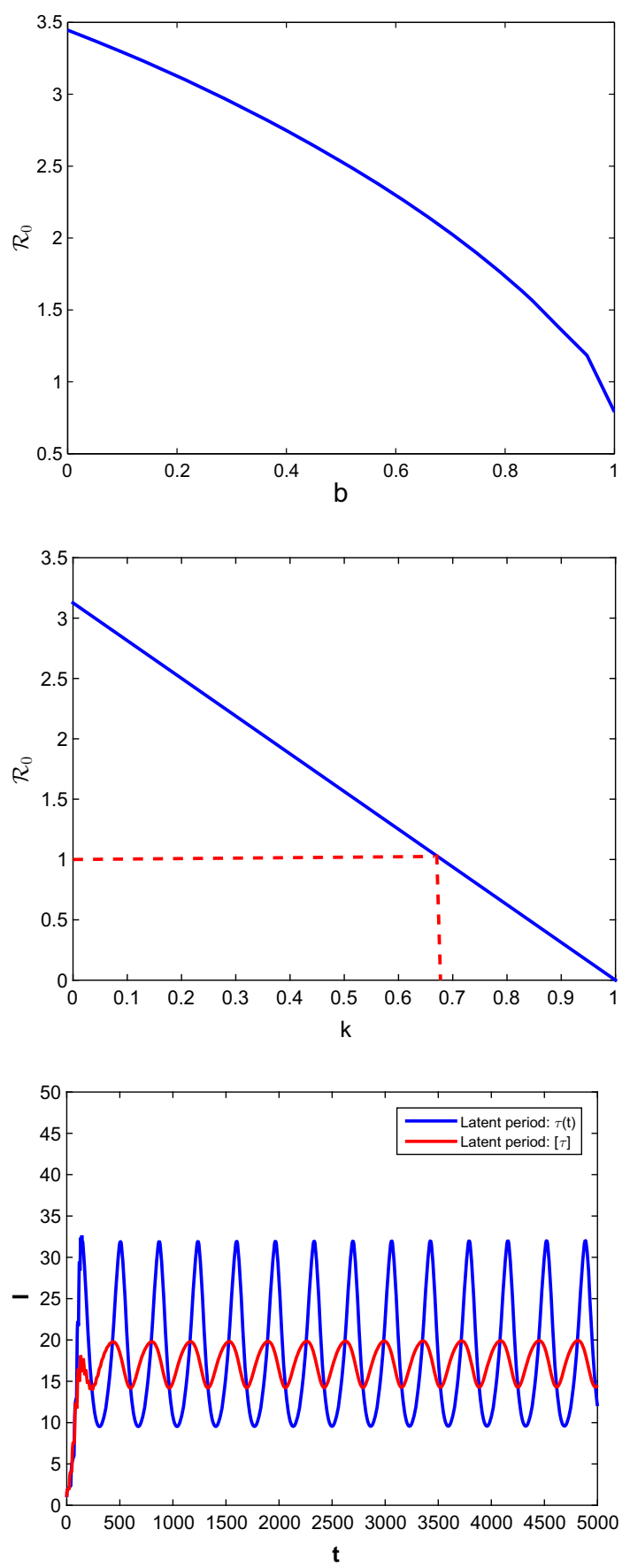
Fig. 8 The basic reproduction ratio $R_{0}$ as a function of $a$ for system (5) with $\tau(t)$ and [ $\tau]$ when $b=0.2$

Fig. 9 The basic reproduction ratio $R_{0}$ as a function of $b$ for system (5) with $\tau(t)$ and $[\tau]$ when $a=0.0015$
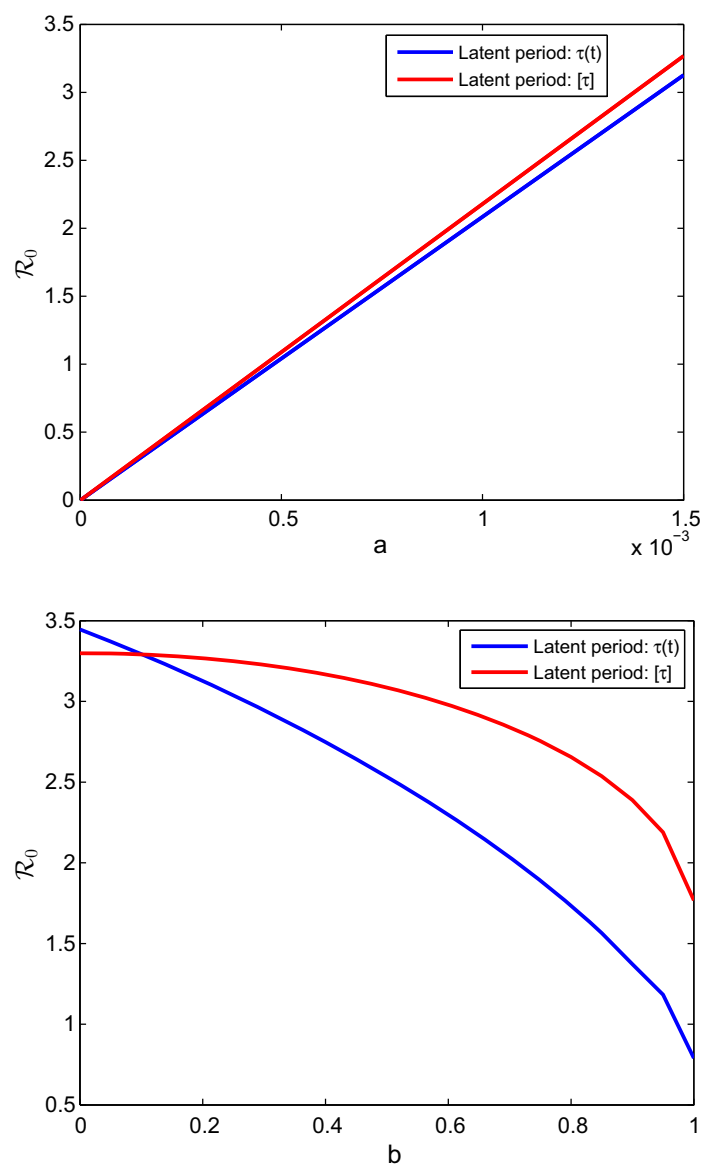

Thirdly, we define the time-averaged latent period as

$$
[\tau]:=\frac{1}{\omega} \int_{0}^{\omega} \tau(t) d t .
$$

It follows that $[\tau]=23$ day. Figure 7 compares the long-term behavior of the infectious population of system (5) with different values of the latent period: the periodic $\tau(t)$ and the constant $[\tau]$. Furthermore, fixed $b=0.2$ and $a=0.0015$, Figs. 8 and 9 compare the effect of periodic $\tau(t)$ and constant $[\tau]$ on $R_{0}$ in these parameter values, respectively. This implies that the use of the time-averaged latent period may underestimate or overestimate the value of $R_{0}$.

\section{Discussion}

In this paper, we have proposed a class of periodic SEIRS epidemic models with general incidence rate by incorporating seasonality into the model so that the parameters are 
periodic functions and the time-dependent delay describes the latent period. By using the theory developed in Zhao (2017a), we have derived the basic reproduction ratio $R_{0}$ for our model system. Following the theoretical approach in Lou and Zhao (2017), we define a new phase space on which the periodic linear system (9) generates an eventually strongly monotone periodic semiflow. By applying the theorem of uniform persistence for periodic semiflows, we have obtained that the basic reproduction ratio $R_{0}$ acts as a threshold parameter for the uniform persistence and global extinction of the disease. If $R_{0}<1$, then the disease-free periodic solution is globally attractive and the disease will be eliminated. If $R_{0}>1$, then there is a positive periodic solution, and the disease is uniformly persistent and exhibits seasonal fluctuations.

For periodic models with time-dependent delays, the numerical approximation of $R_{0}$ is challenging. We have numerically calculated $R_{0}$ and explored the influences of some key parameters in system (5) on $R_{0}$. The numerical simulation about the long-term behavior of solutions is consistent with the obtained analytic results. We have observed that the increase of the transmission coefficient has a negative impact for disease eradication. Therefore, we should make some measures to control disease through decreasing the transmission coefficient for certain parameters.

Furthermore, we have found that there is a difference of the values of $R_{0}$ between the use of $\tau(t)$ and its average $[\tau]$. Using the time-averaged latent period may underestimate or overestimate the value of $R_{0}$. Therefore, in order to find more effective preventive measures during an outbreak of disease, the time-dependent latent delay is important to be considered for some seasonal infectious diseases. Obviously, it is important to acquire some epidemiologically realistic data and to investigate sensitivity studies for the parameters. From the practical viewpoint, our proposed periodic SEIRS epidemic model may be used to understand and predict the outbreak of seasonal infectious diseases.

A possible extension of our model is to consider the case where the incidence rate also depends on the total population size $N(t)$ (see, e.g., Mateus and Silva 2017). As such, the incidence function is of the form $f(t, S(t), I(t), N(t))$, which makes the mathematical analysis of the resulting model more challenging. We leave this interesting problem for future investigation.

Acknowledgements This work was supported in part by the China Scholarship Council (201506460020) and the Natural Science and Engineering Research Council of Canada. We are grateful to two referees for their valuable comments and suggestions which led to an improvement of our original manuscript.

\section{References}

Altizer S, Dobson A, Hosseini P, Hudson P, Pascual M, Rohani P (2006) Seasonality and the dynamics of infectious diseases. Ecol Lett 9(4):467-484

Anderson RM, May RM (1979) Population biology of infectious diseases I. Nature 280:361-367

Aron JL, Schwartz IB (1984) Seasonality and period-doubling bifurcations in an epidemic model. J Theor Biol 110(4):665-679

Beck-Johnson LM, Nelson WA, Paaijmans KP, Read AF, Thomas MB, Bjørnstad ON (2013) The effect of temperature on Anopheles mosquito population dynamics and the potential for malaria transmission. PLoS ONE 8(11):e79276 
Cooke KL, van den Driessche P (1996) Analysis of an SEIRS epidemic model with two delays. J Math Biol 35:240-260

Dowell SF (2001) Seasonal variation in host susceptibility and cycles of certain infectious diseases. Emerg Infect Dis 7(3):369-374

Dowell SF, Whitney CG, Wright C, Rose CE Jr, Schuchat A (2003) Seasonal patterns of invasive pneumococcal disease. Emerg Infect Dis 9:573-579

Fares A (2011) Seasonality of tuberculosis. J Glob Infect Dis 3(1):46-55

Fisman DN (2007) Seasonality of infectious diseases. Annu Rev Public Health 28:127-143

Gao LQ, Mena-Lorca J, Hethcote HW (1995) Four SEI endemic models with periodicity and separatrices. Math Biosci 128(1-2):157-184

Grassly NC, Fraser C (2006) Seasonal infectious disease epidemiology. Proc Biol Sci 273(1600):2541-2550

Greenman J, Kamo M, Boots M (2004) External forcing of ecological and epidemiological systems: a resonance approach. Physica D Nonlinear Phenom 190(1-2):136-151

Groberg WJ, McCoy RH, Pilcher KS, Fryer JL (1978) Relation of water temperature to infections of Coho Salmon (Oncorhynchus kisutch), Chinook Salmon (O. tshawytscha) and Steelhead Trout (Salmo gairdneri) with Aeromonas salmonicida and A. hydrophila. J Fish Res Board Can 35(1):1-7

Hale JK, Verduyn Lunel SM (1993) Introduction to functional differential equations. Springer, New York Hethcote H (1976) Qualitative analyses of communicable disease models. Math Biosci 28:335-356

Huang G, Takeuchi Y, Ma W, Wei D (2010) Global stability for delay SIR and SEIR epidemic models with nonlinear incidence rate. Bull Math Biol 72(5):1192-1207

Jiao J, Chen L, Cai S (2008) An SEIRS epidemic model with two delays and pulse vaccination. J Syst Sci Complex 21:217-225

Kermack WO, McKendrick AG (1932) Contributions to the mathematical theory of epidemics II-the problem of endemicity. Proc R Soc A 138:55-83

Kermack WO, McKendrick AG (1991) Contributions to the mathematical theory of epidemics I. Bull Math Biol 53:33-55

Kot M (2001) Elements of mathematical ecology. Cambridge University Press, New York

Liang X, Zhao X-Q (2007) Asymptotic speeds of spread and traveling waves for monotone semiflows with applications. Commun Pure Appl Math 60:1-40

Liang X, Zhang L, Zhao X-Q (2017) Basic reproduction ratios for periodic abstract functional differential equations (with application to a spatial model for Lyme disease). J Dyn Differ Equ. https://doi.org/ 10.1007/s10884-017-9601-7

Liu L, Zhao X-Q, Zhou Y (2010) A Tuberculosis model with seasonality. Bull Math Biol 72(4):931-952

London WP, Yorke JA (1973) Recurrent outbreaks of measles, chickenpox and mumps. I. Seasonal variation in contact rates. Am J Epidemiol 98(6):453-468

Lou Y, Zhao X-Q (2017) A theoretical approach to understanding population dynamics with seasonal developmental durations. J Nonlinear Sci 27(2):573-603

Lovell DJ, Hunter T, Powers SJ, Parker SR, van den Bosch F (2004) Effect of temperature on latent period of septoria leaf blotch on winter wheat under outdoor conditions. Plant Pathol 53:170-181

Ma W, Song M, Takeuchi Y (2004) Global stability of an SIR epidemic model with time delay. Appl Math Lett 17(10):1141-1145

Mateus JP, Silva CM (2017) Existence of periodic solutions of a periodic SEIRS model with general incidence. Nonlinear Anal Real World Appl 34:379-402

Meyer FP, Warren JW, Carey TG (1983) A guide to integrated fish health management in the Great Lakes basin. Great Lakes Fishery Commission, Ann Arbor, MI. Special Publication, 83-2: 272p

Nakata Y, Kuniya T (2010) Global dynamics of a class of SEIRS epidemic models in a periodic environment. J Math Anal Appl 363(1):230-237

Nisbet RM, Gurney WSC (1983) The systematic formulation of population models for insects with dynamically varying instar duration. Theor Popul Biol 23(1):114-135

Omori R, Adams B (2011) Disrupting seasonality to control disease outbreaks: the case of koi herpes virus. J Theor Biol 271:159-165

Purse BV, Mellor PS, Rogers DJ, Samuel AR, Mertens PP, Baylis M (2005) Climate change and the recent emergence of bluetongue in Europe. Nat Rev Microbiol 3(2):171-181

Qi L, Cui J (2013) The stability of an SEIRS model with nonlinear incidence, vertical transmission and time delay. Appl Math Comput 221:360-366

Smith HL (1995) Monotone dynamical systems: an introduction to the theory of competitive and cooperative systems. American Mathematical Society, Providence 
Snieszko SF (1974) The effects of environmental stress on outbreaks of infectious diseases of fishes. J Fish Biol 6(2):197-208

Sultan B, Labadi K, Guégan JF, Janicot S (2005) Climate drives the meningitis epidemics onset in West Africa. PLoS Med 2:43-49

Towers S, Vogt-Geisse K, Zheng Y, Feng Z (2011) Antiviral treatment for pandemic influenza: assessing potential repercussions using a seasonally forced SIR model. J Theor Biol 289:259-268

Walter W (1997) On strongly monotone flows. Ann Pol Math 66:269-274

Wang W (2002) Global behavior of an SEIRS epidemic model with time delays. Appl Math Lett 15:423-428

Wang X, Zhao X-Q (2017a) Dynamics of a time-delayed Lyme disease model with seasonality. SIAM J Appl Dyn Syst 16(2):853-881

Wang X, Zhao X-Q (2017b) A malaria transmission model with temperature-dependent incubation period. Bull Math Biol 79(5):1155-1182

Vaidya NK, Wahl LM (2015) Avian influenza dynamics under periodic environmental conditions. SIAM J Appl Math 75(2):443-467

Zhang T, Teng Z (2007) On a nonautonomous SEIRS model in epidemiology. Bull Math Biol 69(8):25372559

Zhao X-Q (2017a) Basic reproduction ratios for periodic compartmental models with time delay. J Dyn Differ Equ 29(1):67-82

Zhao X-Q (2017b) Dynamical systems in population biology, 2nd edn. Springer, New York

Publisher's Note Springer Nature remains neutral with regard to jurisdictional claims in published maps and institutional affiliations. 